\title{
ELEMENTARY PROOFS OF ONE WEIGHT NORM INEQUALITIES FOR FRACTIONAL INTEGRAL OPERATORS AND COMMUTATORS
}

\author{
DAVID CRUZ-URIBE, SFO
}

Dedicated to the memory of Professor Cora Sadosky

\begin{abstract}
We give new and elementary proofs of one weight norm inequalities for fractional integral operators and commutators. Our proofs are based on the machinery of dyadic grids and sparse operators used in the proof of the $A_{2}$ conjecture.
\end{abstract}

\section{INTRODUCTION}

The fractional integral operators, also called the Riesz potentials, are the convolution operators

$$
I_{\alpha} f(x)=\int_{\mathbb{R}^{n}} \frac{f(y)}{|x-y|^{n-\alpha}} d y, \quad 0<\alpha<n .
$$

These operators are classical and for $1<p<\frac{n}{\alpha}$ and $q$ defined by $\frac{1}{p}-\frac{1}{q}=\frac{\alpha}{n}$, satisfy $I_{\alpha}: L^{p} \rightarrow L^{q}$. When $p=1$ they satisfy the endpoint estimate $I_{\alpha}: L^{1} \rightarrow L^{q, \infty}$. (Cf. Stein [24].) One weight norm inequalities for these operators were first considered by Muckenhoupt and Wheeden [19], who introduced the governing class of weights, $A_{p, q}$. For $1<p<\frac{n}{\alpha}$ and $q$ such that $\frac{1}{p}-\frac{1}{q}=\frac{\alpha}{n}$, a weight (i.e., a non-negative, locally integrable function) $w$ is in $A_{p, q}$ if

$$
[w]_{A_{p, q}}=\sup _{Q}\left(f_{Q} w^{q} d x\right)^{\frac{1}{q}}\left(f_{Q} w^{-p^{\prime}} d x\right)^{\frac{1}{p^{\prime}}}<\infty
$$

where the supremum is taken over all cubes $Q$ in $\mathbb{R}^{n}$. When $p=1, q=\frac{n}{n-\alpha}$, we say $w \in A_{1, q}$ if

$$
[w]_{A_{1, q}}=\sup _{Q} \underset{x \in Q}{\operatorname{ess} \sup }\left(f_{Q} w^{q} d x\right)^{\frac{1}{q}} w(x)^{-1}<\infty .
$$

Date: March 5, 2015.

2010 Mathematics Subject Classification. 42B25, 42B30, $42 \mathrm{~B} 35$.

Key words and phrases. fractional integral operators, commutators, dyadic operators, weights.

The author is supported by the Stewart-Dorwart faculty development fund at Trinity College and by NSF grant 1362425 . 
Muckenhoupt and Wheeden showed that when $p>1, I_{\alpha}: L^{p}\left(w^{p}\right) \rightarrow L^{q}\left(w^{q}\right)$ if and only $w \in A_{p, q}$, and when $p=1, I_{\alpha}: L^{1}(w) \rightarrow L^{q, \infty}\left(w^{q}\right)$ when $w \in A_{1, q}$. Their proof used a good- $\lambda$ inequality relating $I_{\alpha}$ and the fractional maximal operator,

$$
M_{\alpha} f(x)=\sup _{Q}|Q|^{\frac{\alpha}{n}} f_{Q}|f(y)| d y \cdot \chi_{Q}(x) .
$$

Weighted norm inequalities for $M_{\alpha}$ were proved by generalizing the earlier results for the Hardy-Littlewood maximal operator. A different proof of the strong type inequality was given in [7]: there they used Rubio de Francia extrapolation to prove a norm inequality relating $I_{\alpha}$ and $M_{\alpha}$.

Given $b \in B M O$ we define the commutator of a fractional integral by

$$
\left[b, I_{\alpha}\right] f(x)=b(x) I_{\alpha} f(x)-I_{\alpha}(b f)(x)=\int_{\mathbb{R}^{n}}(b(x)-b(y)) \frac{f(y)}{|x-y|^{n-\alpha}} d y .
$$

These commutators were introduced by Chanillo [2], who proved that with $p$ and $q$ defined as above, $\left[b, I_{\alpha}\right]: L^{p} \rightarrow L^{q}$. He also proved that when $p$ is an even integer, $b \in B M O$ is a necessary condition. (The necessity for the full range of $p$ was recently shown by Chaffee [1].) Commutators are more singular than the fractional integral operator: this can be seen by the fact that when $p=1$, the do not map $L^{1}$ into $L^{q, \infty}$. For a counter-example and substitute endpoint estimate, see [6]. In this paper it was also shown that the strong type inequality is governed by $A_{p, q}$ weights: if $1<p<\frac{n}{\alpha}$ and $w \in A_{p, q}$, then $\left[b, I_{\alpha}\right]: L^{p}\left(w^{p}\right) \rightarrow L^{q}\left(w^{q}\right)$. This proof relied on a sharp maximal function estimate relating the commutator, $I_{\alpha}$, and $M_{\alpha}$. A different proof using extrapolation to relate the commutator to an Orlicz fractional maximal operator was given in [7]. Yet another proof, one that gave the sharp constant in terms of the $[w]_{A_{p, q}}$ characteristic, was given in [9]. This proof used a Cauchy integral formula argument due to Chung, et al. [4].

In this paper we give new and elementary proofs of the one weight inequalities for fractional integral operators and commutators. More precisely, we prove the following three theorems.

Theorem 1.1. Given $0<\alpha<n$, let $q=\frac{n}{n-\alpha}$. If $w \in A_{1, q}$, then for all $f \in L^{1}(w)$,

$$
\sup _{t>0} t w^{q}\left(\left\{x \in \mathbb{R}^{n}:\left|I_{\alpha} f(x)\right|>t\right\}\right)^{\frac{1}{q}} \leq C(n, \alpha)[w]_{A_{1, q}}^{1+q} \int_{\mathbb{R}^{n}}|f(x)| w(x) d x .
$$

Theorem 1.2. Given $0<\alpha<n, 1<p<\frac{n}{\alpha}$, let $q$ be such that $\frac{1}{p}-\frac{1}{q}=\frac{\alpha}{n}$. If $w \in A_{p, q}$, then for all $f \in L^{p}\left(w^{p}\right)$,

$$
\left(\int_{\mathbb{R}^{n}}\left|I_{\alpha} f(x)\right|^{q} w(x)^{q} d x\right)^{\frac{1}{q}} \leq C(n, p, \alpha)[w]_{A_{p, q}}^{1+\frac{q}{p^{\prime}}+\frac{p^{\prime}}{p}}\left(\int_{\mathbb{R}^{n}}|f(x)|^{p} w(x)^{p} d x\right)^{\frac{1}{p}}
$$


Theorem 1.3. Given $0<\alpha<n, 1<p<\frac{n}{\alpha}$, let $q$ be such that $\frac{1}{p}-\frac{1}{q}=\frac{\alpha}{n}$. If $w \in A_{p, q}$, then for all $f \in L^{p}\left(w^{p}\right)$ and $b \in B M O$,

$$
\begin{aligned}
& \left(\left.\int_{\mathbb{R}^{n}}\left|\left[b, I_{\alpha}\right] f(x)\right|\right|^{q} w(x)^{q} d x\right)^{\frac{1}{q}} \\
& \quad \leq C(n, p, \alpha)[w]_{A_{p, q}}^{\max \left(p^{\prime}, q\right)+1+\frac{q}{p^{\prime}}+\frac{p^{\prime}}{p}}\|b\|_{B M O}\left(\int_{\mathbb{R}^{n}}|f(x)|^{p} w(x)^{p} d x\right)^{\frac{1}{p}} .
\end{aligned}
$$

We prove Theorems 1.1-1.3 using the machinery of dyadic grids and sparse operators. Dyadic fractional integral operators date back to the work of Sawyer and Wheeden [23]. More recently, using the machinery developed as part of the proof of the $A_{2}$ conjecture for singular integral operators (see $[15,18]$ and the references they contain) dyadic fractional integral operators were further developed and applied to commutators in $[9,10,11]$. The advantage of this approach is its simplicity: it avoids extrapolation, good- $\lambda$ inequalities and comparisons to the fractional maximal operator. One weakness of our proofs is that they do not give sharp dependence on the $A_{p, q}$ characteristic of the weights: this is to be expected since we freely use their properties to simplify the proofs, whereas any sharp constant proof must be arranged to use their properties as few times as possible. Sharp constants for the fractional integral operator are given in [17], and for commutators in [9].

The remainder of this paper is organized as follows. In Section 2 we give some preliminary results about dyadic grids, sparse operators, weighted fractional maximal operators, and weights. In Section 3 we prove Theorem 1.1. Our proof adapts ideas first used by Sawyer to prove two weight weak $(p, q)$ inequalities for fractional integrals [22]. In Section 4 we prove Theorem 1.2. Our proof uses ideas of Pérez [20] from his proof of two weight inequalities for fractional integrals, and from the elementary proof of one weight inequalities for the Hardy-Littlewood maximal operator due to Christ and Fefferman [3]. In Section 5 we prove Theorem 1.3. Our proof uses some ideas from the proof of two weight results in [9] to reduce the problem to an estimate essentially the same as the one for the fractional integral in the previous section. Finally, in Section 6 we give some personal recollections about the late Cora Sadosky.

Throughout this paper notation is standard or will be defined as needed. The constant $n$ will always denote the dimension. We will denote constants by $C, c$, etc. and their value may change at each appearance. Unless otherwise specified, we will assume that constants can depend on $p, \alpha$ and $n$ but we will keep track of the dependence on the $A_{p, q}$ characteristic explicitly. 


\section{Preliminary Results}

Dyadic grids and operators. We begin by defining dyadic grids and the dyadic fractional integral operators. Unless otherwise noted, the results given here are taken from $[5,9,10,11]$.

Definition 2.1. A collection of cubes $\mathcal{D}$ in $\mathbb{R}^{n}$ is a dyadic grid if provided that:

(1) If $Q \in \mathcal{D}$, then $\ell(Q)=2^{k}$ for some $k \in \mathbb{Z}$.

(2) If $P, Q \in \mathcal{D}$, then $P \cap Q \in\{P, Q, \emptyset\}$.

(3) For every $k \in \mathbb{Z}$, the cubes $\mathcal{D}_{k}=\left\{Q \in \mathcal{D}: \ell(Q)=2^{k}\right\}$ form a partition of $\mathbb{R}^{n}$.

Definition 2.2. Given a dyadic grid $\mathcal{D}$, a set $\mathcal{S} \subset \mathcal{D}$ is sparse if for every $Q \in S$,

$$
\left|\bigcup_{\substack{P \in S \\ P \subsetneq Q}} P\right| \leq \frac{1}{2}|Q|
$$

Equivalently, if we define

$$
E(Q)=Q \backslash \bigcup_{\substack{P \in S \\ P \subsetneq Q}} P,
$$

then the sets $E(Q)$ are pairwise disjoint and $|E(Q)| \geq \frac{1}{2}|Q|$.

The classic example of a dyadic grid and sparse families are the standard dyadic grid on $\mathbb{R}^{n}$ and the Calderón-Zygmund cubes associated with an $L^{1}$ function. See $[8$, Appendix A].

We next define a dyadic version of the fractional integral operator and show that it can be used to bound $I_{\alpha}$ pointwise. For $f \in L_{l o c}^{1}$ and a cube $Q$, let

$$
\langle f\rangle_{Q}=f_{Q} f(x) d x=\frac{1}{|Q|} \int_{Q} f(x) d y .
$$

Given $0<\alpha<n$ and a dyadic grid $\mathcal{D}$, define

$$
I_{\alpha}^{\mathcal{D}} f(x)=\sum_{Q \in \mathcal{D}}|Q|^{\frac{\alpha}{n}}\langle f\rangle_{Q} \cdot \chi_{Q}(x) .
$$

Similarly, given a sparse subset $\mathcal{S} \subset \mathcal{D}$, define

$$
I_{\alpha}^{\mathcal{S}} f(x)=\sum_{Q \in \mathcal{S}}|Q|^{\frac{\alpha}{n}}\langle f\rangle_{Q} \cdot \chi_{Q}(x) .
$$

Lemma 2.3. There exists a collection $\left\{\mathcal{D}_{i}\right\}_{i=1}^{N}$ of dyadic grids such that for $0<\alpha<n$ and every non-negative function $f$,

$$
I_{\alpha} f(x) \leq C \sup _{i} I_{\alpha}^{\mathcal{D}_{i}} f(x) .
$$


Moreover, given any dyadic grid $\mathcal{D}$ and a non-negative function $f \in L_{c}^{\infty}$, there exists a sparse set $\mathcal{S} \subset \mathcal{D}$ such that

$$
I^{\mathcal{D}} f(x) \leq C I^{\mathcal{S}} f(x) .
$$

Given $b \in B M O$ and $0<\alpha<n$, for any dyadic grid $\mathcal{D}$, define the dyadic commutator

$$
C_{b}^{\mathcal{D}} f(x)=\sum_{Q \in \mathcal{D}}|Q|^{\frac{\alpha}{n}} f_{Q}|b(x)-b(y)| f(y) d y \cdot \chi_{Q}(x) .
$$

Lemma 2.4. There exists a collection $\left\{\mathcal{D}_{i}\right\}_{i=1}^{N}$ of dyadic grids such that for $0<\alpha<$ $n$, every non-negative function $f$, and every $b \in B M O$,

$$
\left|\left[b, I_{\alpha}\right] f(x)\right| \leq C \sup _{i} C_{b}^{\mathcal{D}^{i}} f(x) .
$$

Remark 2.5. It follows at once from Lemmas 2.3 and 2.4 that to prove norm inequalities for $I_{\alpha}$ and $\left[b, I_{\alpha}\right]$ it will suffice to prove them for their dyadic counterparts. Moreover, since these dyadic integral operators are positive, we may assume that $f$ is non-negative in our proofs. Finally, by Fatou's lemma it will suffice to prove our results for functions $f \in L_{c}^{\infty}$. In particular, this will let us pass to sparse operators.

Weighted fractional maximal operators. We begin with some basic facts about Orlicz spaces. Some of these will also be needed in Section 5 below. For further information on these spaces, see [21]; for their use in weighted norm inequalities, see [8]. Given a weight $\sigma$, let $d \sigma=\sigma d x$. We define averages with respect to the measure $d \sigma$ :

$$
\langle f\rangle_{Q, \sigma}=f_{Q} f(x) d \sigma=\frac{1}{\sigma(Q)} \int_{Q} f(x) d \sigma .
$$

Given a Young function $\Phi$ and a cube $Q$, define the normalized Luxemburg norm with respect to $\Phi$ and $d \sigma$ by

$$
\|f\|_{\Phi, Q, \sigma}=\inf \left\{\lambda>0: f_{Q} \Phi\left(\frac{|f(x)|}{\lambda}\right) d \sigma \leq 1\right\} .
$$

If we let $\Phi(t)=t^{p}, 1 \leq p<\infty$, then

$$
\|f\|_{\Phi, Q, \sigma}=\left(f_{Q}|f(x)|^{p} d \sigma\right)^{\frac{1}{p}}=\|f\|_{p, Q, \sigma} .
$$

Associated to any Young function is its associate function $\bar{\Phi}$. We have the generalized Hölder's inequality: for any cube $Q$,

$$
f_{Q}|f(x) g(x)| d \sigma \leq C\|f\|_{\Phi, Q, \sigma}\|g\|_{\bar{\Phi}, Q, \sigma} ;
$$

the constant depends only on $\Phi$. 
Hereafter, we will let $\Phi(t)=t \log (e+t)$; then it can be shown that $\bar{\Phi}(t) \approx e^{t}-1$. It follows for this choice of $\Phi$ that for $1<p<\infty$,

$$
\|f\|_{1, Q, \sigma} \leq\|f\|_{\Phi, Q, \sigma} \leq C(p)\|f\|_{p, Q, \sigma} .
$$

We now define a weighted dyadic fractional maximal operator. Given a dyadic grid $\mathcal{D}$ and a weight $\sigma$, for $0 \leq \alpha<n$ define

$$
M_{\Phi, \sigma, \alpha}^{\mathcal{D}} f(x)=\sup _{Q \in \mathcal{D}} \sigma(Q)^{\frac{\alpha}{n}}\|f\|_{\Phi, Q, \sigma}
$$

If $\Phi(t)=t$ we write $M_{\sigma, \alpha}^{\mathcal{D}}$. If $\alpha=0$, then we simply write $M_{\Phi, \sigma}^{\mathcal{D}}$ or $M_{\sigma}^{\mathcal{D}}$ if $\Phi(t)=t$.

Lemma 2.6. Let $\Phi(t)=t \log (e+t)$. Given $1<p<\infty$ and $0 \leq \alpha<n$, define $q$ by $\frac{1}{p}-\frac{1}{q}=\frac{\alpha}{n}$. Given a weight $\sigma$ and a dyadic grid $\mathcal{D}, M_{\Phi, \sigma, \alpha}^{\mathcal{D}}: L^{p}(\sigma) \rightarrow L^{q}(\sigma)$. The same inequality holds for $M_{\sigma, \alpha}^{\mathcal{D}}$.

Proof. This result is well-known when $\Phi(t)=t$; the proof is essentially the same for $\Phi(t)=t \log (e+t)$ and we sketch the details. By off-diagonal Marcinkiewicz interpolation (see [25]) it will suffice to prove the corresponding weak $(p, q)$ inequality:

$$
\sigma\left(\left\{x \in \mathbb{R}^{n}: M_{\Phi, \sigma, \alpha}^{\mathcal{D}} f(x)>t\right\}\right)^{\frac{1}{q}} \leq \frac{C}{t}\left(\int_{\mathbb{R}^{n}}|f(x)|^{p} d \sigma\right)^{\frac{1}{p}} .
$$

Fix $t>0$; then we can decompose the level set as the union of disjoint cubes $Q \in$ $\mathcal{Q}_{t} \subset \mathcal{D}$ that satisfy

$$
\sigma(Q)^{\frac{\alpha}{n}}\|f\|_{\Phi, Q, \sigma}>t
$$

Therefore, since the cubes in $\mathcal{Q}_{t}$ are disjoint and $q / p \geq 1$, we have that

$$
\begin{aligned}
\sigma\left(\left\{x \in \mathbb{R}^{n}: M_{\Phi, \sigma, \alpha}^{\mathcal{D}} f(x)>t\right\}\right) & =\sum_{Q \in \mathcal{Q}_{t}} \sigma(Q) \\
& \leq t^{-q} \sum_{Q \in \mathcal{Q}_{t}} \sigma(Q)^{1+q \frac{\alpha}{n}}\|f\|_{\Phi, Q, \sigma}^{q} \\
& \leq \frac{C}{t^{q}} \sum_{Q \in \mathcal{Q}_{t}} \sigma(Q)^{1+q \frac{\alpha}{n}}\left(f_{Q}|f|^{p} d \sigma\right)^{\frac{q}{p}} \\
& \leq \frac{C}{t^{q}} \sum_{Q \in \mathcal{Q}_{t}}\left(\int_{Q}|f|^{p} d \sigma\right)^{\frac{q}{p}} \\
& \leq \frac{C}{t^{q}}\left(\sum_{Q \in \mathcal{Q}_{t}} \int_{Q}|f|^{p} d \sigma\right)^{\frac{q}{p}} \\
& \leq \frac{C}{t^{q}}\left(\int_{\mathbb{R}^{n}}|f(x)|^{p} d \sigma\right)^{\frac{q}{p}} .
\end{aligned}
$$


Properties of $A_{p, q}$ weights. In this section we gather a few basic facts about the $A_{p, q}$ weights and the closely related Muckenhoupt $A_{p}$ weights; for further information see [12]. For $1<p<\infty, w \in A_{p}$ if

$$
[w]_{A_{p}}=\sup _{Q} f_{Q} w(x) d x\left(f_{Q} w(x)^{1-p^{\prime}} d x\right)^{p-1}<\infty .
$$

For $p=1, w \in A_{1}$ if

$$
[w]_{A_{1}}=\sup _{Q} \operatorname{ess} \sup (f Q w(x) d x) w(x)^{-1}<\infty .
$$

It follows at once from the definition that for all $p$ and $q$ such that $\frac{1}{p}-\frac{1}{q}=\frac{\alpha}{n}$, then $w \in A_{p, q}$ if and only if $w^{q} \in A_{r}, r=1+\frac{q}{p^{\prime}}$ and $\left[w^{q}\right]_{A_{r}}=[w]_{A_{p, q}}^{q}$. (When $p=1$, we interpret $\frac{q}{p^{\prime}}$ as 0.) By the duality of $A_{p}$ weights, $w^{-p^{\prime}} \in A_{r^{\prime}}$ and $\left[w^{-p^{\prime}}\right]_{A_{r^{\prime}}}=[w]_{A_{p, q}}^{p^{\prime}}$.

As a consequence, we have that both $w^{q}$ and $w^{-p^{\prime}}$ are in $A_{\infty}$. Below we will need to use two properties of $A_{\infty}$ weights; for both we give the sharp constant version. There are multiple definitions of the $A_{\infty}$ characteristic (cf. [13]) but for our purposes we will simply use the fact that $[w]_{A_{\infty}} \leq C(n)[w]_{A_{p}}$.

Lemma 2.7. Given a weight $\sigma \in A_{p} \subset A_{\infty}$, then for any cube $Q$ and set $E \subset Q$ :

(1) $\left(\frac{|E|}{|Q|}\right)^{p} \leq[\sigma]_{A_{p}} \frac{\sigma(E)}{\sigma(Q)}$;

(2) $\frac{\sigma(E)}{\sigma(Q)} \leq 2\left(\frac{|E|}{|Q|}\right)^{\frac{1}{s^{\prime}}}$, where $s^{\prime}=c(n)[\sigma]_{A_{\infty}}$.

Proof. The first inequality follows from the definition of $A_{p}$ : see [12]. The second follows from the sharp form of the reverse Hölder inequality due to Hytönen and Pérez [16]: for every cube $Q$,

$$
\left(f_{Q} \sigma(x)^{s} d x\right)^{\frac{1}{s}} \leq 2 f_{Q} \sigma(x) d x
$$

where $s=1+\frac{1}{c(n)[\sigma]_{A_{\infty}}}$. The desired inequality follows if we apply Hölder's inequality with exponent $s$ to $f_{Q} \sigma(x) \chi_{E}(x) d x$.

\section{Proof of Theorem 1.1}

By Remark 2.5 it will suffice to prove the weak $(1, q)$ inequality for the dyadic operator $I_{\alpha}^{\mathcal{D}}$, where $\mathcal{D}$ is an arbitrary dyadic grid, and for $f$ a non-negative function in $L_{c}^{\infty}$. Furthermore, since $\operatorname{supp}(f)$ is contained in the union of at most $2^{n}$ cubes in 
$\mathcal{D}$ (of equal size), by linearity it will suffice to prove this inequality assuming $\operatorname{supp}(f)$ is contained in a single cube.

Given a cube $Q$, we decompose $I_{\alpha}^{\mathcal{D}}$ into its inner and outer parts:

$$
\begin{aligned}
I_{\alpha}^{\mathcal{D}} f(x)=\sum_{P \in \mathcal{D}}|P|^{\frac{\alpha}{n}}\langle f\rangle_{P} \chi_{P}(x) & =\sum_{\substack{P \in \mathcal{D} \\
P \subseteq Q}}|P|^{\frac{\alpha}{n}}\langle f\rangle_{P} \chi_{P}(x)+\sum_{\substack{P \in \mathcal{D} \\
Q \subseteq P}}|P|^{\frac{\alpha}{n}}\langle f\rangle_{P} \chi_{P}(x) \\
& =I_{\alpha, Q}^{\mathcal{D}, \text { in }} f(x)+I_{\alpha, Q}^{\mathcal{D}, \text { out }} f(x) .
\end{aligned}
$$

For each $t>0$ define the set

$$
E_{t}=\left\{x \in \mathbb{R}^{n}: I_{\alpha}^{\mathcal{D}} f(x)>t\right\} .
$$

We can write $E_{t}$ as the union of a collection $\mathcal{Q}_{t}$ of maximal, disjoint cubes in $\mathcal{D}$. By maximality, if $Q \in \mathcal{Q}_{t}$ and we let $\hat{Q}$ be its dyadic parent, then there exists $z \in \hat{Q} \backslash Q$ such that for every $x \in Q$,

$$
t \geq I_{\alpha}^{D} f(z) \geq I_{\alpha, Q}^{\mathcal{D}, \text { out }} f(z)=I_{\alpha, Q}^{\mathcal{D}, \text { out }} f(x) .
$$

In particular, if we take any $x \in Q \cap E_{2 t}$, then we must have that

$$
I_{\alpha, Q}^{\mathcal{D}, i n} f(x)>t
$$

Now fix $T>0$ and let $t$ be such that $0<t<T / 2$. Let $v=w^{q}$. Partition the cubes in $\mathcal{Q}_{t}$ into two sets: we say $Q \in \mathcal{Q}_{L}$ (large cubes) if

$$
v\left(Q \cap E_{2 t}\right) \geq 2^{-q-1} v(Q),
$$

and if the reverse inequality holds we say $Q \in \mathcal{Q}_{S}$ (small cubes). Since $E_{2 t} \subset E_{t}$, we have that

$$
\begin{aligned}
(2 t)^{q} v\left(E_{2 t}\right) & =(2 t)^{q} \sum_{Q \in \mathcal{Q}_{t}} v\left(Q \cap E_{2 t}\right) \\
& =(2 t)^{q} \sum_{Q \in \mathcal{Q}_{S}} v\left(Q \cap E_{2 t}\right)+(2 t)^{q} \sum_{Q \in \mathcal{Q}_{L}} v\left(Q \cap E_{2 t}\right) \\
& =I_{S}+I_{L} .
\end{aligned}
$$

We estimate each sum separately. The first is straightforward:

$$
I_{S} \leq(2 t)^{q} \sum_{Q \in \mathcal{Q}_{S}} 2^{-q-1} v(Q)=\frac{1}{2} t^{q} v\left(E_{t}\right) \leq \frac{1}{2} \sup _{0<t<T / 2} t^{q} v\left(E_{t}\right) \leq \frac{1}{2} \sup _{0<t<T} t^{q} v\left(E_{t}\right) .
$$

To estimate the second, by inequality (3.1) and duality (since $I_{\alpha, Q}^{\mathcal{D}}$ is self-adjoint) we have that

$$
I_{L}=(2 t)^{q} \sum_{Q \in \mathcal{Q}_{L}} v\left(Q \cap E_{t}\right)^{q} v\left(Q \cap E_{t}\right)^{1-q}
$$




$$
\begin{aligned}
& \leq(2 t)^{q} \sum_{Q \in \mathcal{Q}_{L}} v\left(\left\{x \in Q: I_{\alpha, Q}^{\mathcal{D}, i n} f(x)>t\right\}\right)^{q} v\left(Q \cap E_{t}\right)^{1-q} \\
& \leq C(q) \sum_{Q \in \mathcal{Q}_{L}}\left(\int_{Q} I_{\alpha, Q}^{\mathcal{D}, i n} f(x) v(x) d x\right)^{q} v(Q)^{1-q} \\
& =C(q) \sum_{Q \in \mathcal{Q}_{L}}\left(\int_{Q} f(x) I_{\alpha, Q}^{\mathcal{D}, i n} v(x) d x\right)^{q} v(Q)^{1-q}
\end{aligned}
$$

By the definition of $I_{\alpha, Q}^{\mathcal{D}, \text { in }}$ and $q$,

$$
I_{\alpha, Q}^{\mathcal{D}, \text { in }} v(x)=\sum_{\substack{P \in \mathcal{D} \\ P \subseteq Q}}|P|^{\frac{\alpha}{n}}\langle v\rangle_{P} \chi_{P}(x)=\sum_{\substack{P \in \mathcal{D} \\ P \subseteq Q}}|P|^{\frac{1}{q^{\prime}}}\langle v\rangle_{P}^{\frac{1}{q^{\prime}}}\langle v\rangle_{P}^{\frac{1}{q}} \chi_{P}(x)
$$

Since $w \in A_{1, q}, v \in A_{1} \subset A_{\infty}$, and so for any $P \subseteq Q\langle v\rangle_{P}^{\frac{1}{q}} \leq[w]_{A_{1, q}} w(x)$, and by Lemma 2.7,

$$
v(P) \leq 2\left(\frac{|P|}{|Q|}\right)^{\frac{1}{s^{\prime}}} v(Q)
$$

where $s^{\prime} \leq c(n)[w]_{1, q}^{q}$. If we fix $x$, then there exists a nested sequence of dyadic cubes $P_{k} \subset Q, k \geq 0$, such that $x \in P_{k}$ and $\ell\left(P_{k}\right)=2^{-k} \ell(Q)$. Therefore,

$$
\sum_{\substack{P \in \mathcal{D} \\ P \subseteq Q}}|P|^{\frac{1}{q^{\prime}}}\langle v\rangle_{P}^{\frac{1}{q^{\prime}}} \chi_{P}(x)=\sum_{k=0}^{\infty} v\left(P_{k}\right)^{\frac{1}{q^{\prime}}} \leq 2^{\frac{1}{q^{\prime}}} \sum_{k=0}^{\infty} 2^{-\frac{k n}{q^{\prime} s^{\prime}}} v(Q)^{\frac{1}{q^{\prime}}} \leq C s^{\prime} v(Q)^{\frac{1}{q^{\prime}}} .
$$

If we combine all of these estimates we get that

$$
I_{\alpha, Q}^{\mathcal{D}, i n}(v)(x) \lesssim[w]_{A_{1, q}}^{1+q} v(Q)^{\frac{1}{q^{\prime}}} w(x) .
$$

Hence,

$$
\begin{aligned}
I_{L} \leq C[w]_{A_{1, q}}^{q(q+1)} \sum_{Q \in \mathcal{Q}_{L}} & \left(\int_{Q} f(x) w(x) d x\right)^{q} v(Q)^{\frac{q}{q^{\prime}}+1-q} \\
& \leq[w]_{A_{1, q}}^{q(q+1)}\left(\sum_{Q \in \mathcal{Q}_{L}} \int_{Q} f(x) w(x) d x\right)^{q} \leq[w]_{A_{1, q}}^{q(q+1)}\left(\int_{\mathbb{R}^{n}} f(x) w(x) d x\right)^{q} .
\end{aligned}
$$

If we combine this with the estimate for $I_{S}$, we get

$$
(2 t)^{q} v\left(E_{2 t}\right) \leq \frac{1}{2} \sup _{0<t<T} t^{q} v\left(E_{t}\right)+C[w]_{A_{1, q}}^{q(q+1)}\left(\int_{\mathbb{R}^{n}} f(x) w(x) d x\right)^{q} .
$$


Since this inequality holds for any $t, 0<t<T / 2$, on the left, we can take the supremum over these values of $t$ and rearrange terms to get

$$
\sup _{0<t<T} t^{q} v\left(E_{t}\right) \leq C[w]_{A_{1, q}}^{q(q+1)}\left(\int_{\mathbb{R}^{n}} f(x) w(x) d x\right)^{q}
$$

provided that the supremum is finite. The desired inequality would then follow if we let $T \rightarrow \infty$.

To see that this supremum is finite: By assumption, $\operatorname{supp}(f) \subset Q$ for some cube $Q \in \mathcal{D}$. If $x \notin Q$, then $I_{\alpha}^{\mathcal{D}} f(x) \neq 0$, only if there exists a dyadic cube $P$ that contains $x$ and $Q$. Let $P_{0}$ be the smallest such cube, and let $\left\{P_{k}\right\}$ be the sequence of dyadic cubes containing $x$ such that $P_{0} \subset P_{k}$ and $\ell\left(P_{k}\right)=2^{k} \ell\left(P_{0}\right)$. Then

$$
I_{\alpha}^{\mathcal{D}} f(x)=\sum_{k=0}^{\infty} 2^{k(\alpha-n)}\left|P_{0}\right|^{\frac{\alpha}{n}-1} \int_{P_{k}} f(y) d y \leq C\left|P_{0}\right|^{\frac{\alpha}{n}-1} \int_{P_{0}} f(y) d y \leq C M_{\alpha}^{\mathcal{D}} f(x) .
$$

Therefore, we have that

$$
\sup _{0<t<T} t^{q} v\left(E_{t}\right) \leq T^{q} v(Q)+\sup _{0<t<T} t^{q} v\left(\left\{x \in \mathbb{R}^{n} \backslash Q: M_{\alpha}^{\mathcal{D}} f(x)>t / C\right\}\right) .
$$

The first term is finite since $v$ is locally integrable, and the second is finite by the weighted weak $(1, q)$ inequality for $M_{\alpha}^{\mathcal{D}}$ (cf. [19]). This completes the proof.

\section{Proof of TheOrem 1.2}

By Remark 2.5, it will suffice to prove the strong $(p, q)$ inequality for $f$ non-negative and in $L_{c}^{\infty}$. We may also replace $I_{\alpha}$ by the sparse operator $I_{\alpha}^{\mathcal{S}}$, where $\mathcal{S}$ is any sparse subset of a dyadic grid $\mathcal{D}$.

Let $v=w^{q}$ and $\sigma=w^{-p^{\prime}}$ and estimate as follows: there exists $g \in L^{q^{\prime}}\left(w^{-q^{\prime}}\right)$, $\left\|g w^{-1}\right\|_{q^{\prime}}=1$, such that

$$
\begin{aligned}
\left\|\left(I_{\alpha}^{\mathcal{S}} f\right) w\right\|_{q}=\int_{\mathbb{R}^{n}} I_{\alpha} f(x) g(x) & d x=\sum_{Q \in \mathcal{S}}|Q|^{\frac{\alpha}{n}}\langle f\rangle_{Q} \int_{Q} g(x) d x \\
= & \sum_{Q \in \mathcal{S}}|Q|^{\frac{\alpha}{n}-1} \sigma(Q) v(Q)^{1-\frac{\alpha}{n}}\left\langle f \sigma^{-1}\right\rangle_{Q, \sigma} v(Q)^{\frac{\alpha}{n}}\left\langle g v^{-1}\right\rangle_{Q, v} .
\end{aligned}
$$

Since $1-\frac{\alpha}{n}=\frac{1}{p^{\prime}}+\frac{1}{q}$, by the definition of the $A_{p, q}$ condition and Lemma 2.7 (applied to both $v$ and $\sigma$ ) we have that

$$
|Q|^{\frac{\alpha}{n}-1} \sigma(Q) v(Q)^{1-\frac{\alpha}{n}} \leq[w]_{A_{p, q}} \sigma(Q)^{\frac{1}{p}} v(Q)^{\frac{1}{p^{\prime}}} \leq[w]_{A_{p, q}}^{1+\frac{q}{p^{\prime}}+\frac{p^{\prime}}{p}} \sigma(E(Q))^{\frac{1}{p}} v(E(Q))^{\frac{1}{p^{\prime}}}
$$

If we combine these two estimates, then by Hölder's inequality and Lemma 2.6 we get that

$\left\|\left(I_{\alpha}^{\mathcal{S}} f\right) w\right\|_{q}$ 


$$
\begin{aligned}
& \leq C[w]_{A_{p, q}}^{1+\frac{q}{p^{\prime}}+\frac{p^{\prime}}{p}} \sum_{Q \in \mathcal{S}}\left\langle f \sigma^{-1}\right\rangle_{Q, \sigma} \sigma(E(Q))^{\frac{1}{p}} v(Q)^{\frac{\alpha}{n}}\left\langle g v^{-1}\right\rangle_{Q, v} v(E(Q))^{\frac{1}{p^{\prime}}} \\
& \leq C[w]_{A_{p, q}}^{1+\frac{q}{p^{\prime}}+\frac{p^{\prime}}{p}}\left(\sum_{Q \in \mathcal{S}}\left\langle f \sigma^{-1}\right\rangle_{Q, \sigma}^{p} \sigma(E(Q))\right)^{\frac{1}{p}}\left(\sum_{Q \in \mathcal{S}}\left[v(Q)^{\frac{\alpha}{n}}\left\langle g v^{-1}\right\rangle_{Q, v}\right]^{p^{\prime}} v(E(Q))\right)^{\frac{1}{p^{\prime}}} \\
& \leq C[w]_{A_{p, q}}^{1+\frac{q}{p^{\prime}}+\frac{p^{\prime}}{p}}\left(\sum_{Q \in \mathcal{S}} \int_{E(Q)} M_{\sigma}^{\mathcal{D}}\left(f \sigma^{-1}\right)(x)^{p} d \sigma\right)^{\frac{1}{p}}\left(\sum_{Q \in \mathcal{S}} \int_{E(Q)} M_{v, \alpha}^{\mathcal{D}}\left(g v^{-1}\right)(x)^{p^{\prime}} d v\right)^{\frac{1}{p^{\prime}}} \\
& \leq C[w]_{A_{p, q}}^{1+\frac{q}{p^{\prime}}+\frac{p^{\prime}}{p}}\left(\int_{\mathbb{R}^{n}} M_{\sigma}^{\mathcal{D}}\left(f \sigma^{-1}\right)(x)^{p} d \sigma\right)^{\frac{1}{p}}\left(\int_{\mathbb{R}^{n}} M_{v, \alpha}^{\mathcal{D}}\left(g v^{-1}\right)(x)^{p^{\prime}} d v\right)^{\frac{1}{p^{\prime}}} \\
& \leq C[w]_{A_{p, q}}^{1+\frac{q}{p^{\prime}}+\frac{p^{\prime}}{p}}\left(\int_{\mathbb{R}^{n}}\left(f(x) \sigma(x)^{-1}\right)^{p} d \sigma\right)^{\frac{1}{p}}\left(\int_{\mathbb{R}^{n}}\left(g(x) v(x)^{-1}\right)^{q^{\prime}} d v\right)^{\frac{1}{q^{\prime}}} \\
& =C[w]_{A_{p, q}}^{1+\frac{q}{p^{\prime}}+\frac{p^{\prime}}{p}}\|f w\|_{p}\left\|g w^{-1}\right\|_{q^{\prime}} \\
& =C[w]_{A_{p, q}}^{1+\frac{q}{p^{\prime}}+\frac{p^{\prime}}{p}}\|f w\|_{p} .
\end{aligned}
$$

\section{Proof of Theorem 1.3}

For our proof we need two lemmas. The first is a weighted estimate for functions in $B M O$; our proof adapts ideas from Ho [14].

Lemma 5.1. Let $\Phi(t)=t \log (e+t)$. Given a weight $\sigma \in A_{\infty}$, then for any $b \in B M O$ and any cube $Q$,

$$
\left\|b-\langle b\rangle_{Q}\right\|_{\bar{\Phi}, Q, \sigma} \leq C[\sigma]_{A_{\infty}}\|b\|_{B M O} .
$$

Proof. By the John-Nirenberg inequality, there exist constants $C_{1}, C_{2}$ such that for every cube $Q$ and $\lambda, t>0$,

$$
\left|\left\{x \in Q:\left|b(x)-\langle b\rangle_{Q}\right|>\lambda t\right\}\right| \leq C_{1}|Q| \exp \left(-\frac{C_{2} \lambda t}{\|b\|_{B M O}}\right) .
$$

Since $\sigma \in A_{\infty}$, by Lemma 2.7 we have that

$$
\sigma\left(\left\{x \in Q:\left|b(x)-\langle b\rangle_{Q}\right|>\lambda t\right\}\right) \leq 2 C_{1}^{\frac{1}{s^{\prime}}} \sigma(Q) \exp \left(-\frac{C_{2} \lambda t}{\|b\|_{B M O} s^{\prime}}\right),
$$

where $s^{\prime}=c(n)[\sigma]_{A_{\infty}}$. Let

$$
\lambda=\frac{\left(1+2 k C_{1}^{\frac{1}{s^{\prime}}}\right)\|b\|_{B M O} s^{\prime}}{C_{2}}=C[\sigma]_{A_{\infty}}\|b\|_{B M O}
$$


where $\bar{\Phi}(t) \leq k e^{t}$. Then we have that

$$
\begin{aligned}
f_{Q} \bar{\Phi}\left(\frac{\left|b(x)-\langle b\rangle_{Q}\right|}{\lambda}\right) d \sigma & \leq k \sigma(Q)^{-1} \int_{0}^{\infty} e^{t} \sigma\left(\left\{x \in Q:\left|b(x)-\langle b\rangle_{Q}\right|>\lambda t\right\}\right) d t \\
& \leq 2 k C_{1}^{\frac{1}{s^{\prime}}} \int_{0}^{\infty} e^{t} \exp \left(-\frac{C_{2} \lambda t}{\|b\|_{B M O} s^{\prime}}\right) d t \\
& \leq 2 k C_{1}^{\frac{1}{s^{\prime}}} \int_{0}^{\infty} e^{-2 k C_{1}^{\frac{1}{s^{\prime}}} t} d t \\
& =1 .
\end{aligned}
$$

Therefore, by the definition of the Luxemburg norm, we get the desired inequality.

The second lemma is a weighted variant of an estimate from [7]; when $\Phi(t)=t$ the unweighted estimate is originally due to Sawyer and Wheeden [23].

Lemma 5.2. Fix $0<\alpha<n$, a dyadic grid $\mathcal{D}$, a weight $\sigma$ and a Young function $\Phi$. Then for any $P \in \mathcal{D}$ and any function $f$,

$$
\left.\sum_{\substack{Q \in \mathcal{D} \\ Q \subset P}}|Q|^{\frac{\alpha}{n}} \sigma(Q)\left|\|f\|_{\Phi, Q, \sigma} \leq C(\alpha)\right| P\right|^{\frac{\alpha}{n}} \sigma(P) \mid\|f\|_{\Phi, P, \sigma} .
$$

Proof. To prove this we need to replace the Luxemburg norm with the equivalent Amemiya norm [21, Section 3.3]:

$$
\|f\|_{\Phi, P, \sigma} \leq \inf _{\lambda>0}\left\{\lambda f_{P} 1+\Phi\left(\frac{|f(x)|}{\lambda}\right) d \sigma\right\} \leq 2\|f\|_{\Phi, P, \sigma} .
$$

By the second inequality, we can fix $\lambda_{0}>0$ such that the middle quantity is less than $3\|f\|_{\Phi, P, \sigma}$. Then by the first inequality,

$$
\begin{aligned}
\sum_{\substack{Q \in \mathcal{D} \\
Q \subset P}}|Q|^{\frac{\alpha}{n}}|Q|\|f\|_{\Phi, Q, \sigma} & =\sum_{k=0}^{\infty} \sum_{\substack{Q \subset P \\
\ell(Q)=2^{-k} \ell(P)}}|Q|^{\frac{\alpha}{n}}|Q|\|f\|_{\Phi, Q, \sigma} \\
& \leq|P|^{\frac{\alpha}{n}} \sum_{k=0}^{\infty} 2^{-k \alpha} \sum_{\substack{Q \subset P \\
\ell(Q)=2^{-k} \ell(P)}} \lambda_{0} \int_{Q} 1+\Phi\left(\frac{|f(x)|}{\lambda_{0}}\right) d \sigma \\
& =C|P|^{\frac{\alpha}{n}} \lambda_{0} \int_{P} 1+\Phi\left(\frac{|f(x)|}{\lambda_{0}}\right) d \sigma \\
& \leq C|P|^{\frac{\alpha}{n}} \sigma(P)\|f\|_{\Phi, P, \sigma} .
\end{aligned}
$$


Proof of Theorem 1.3. Again by Remark 2.5, it will suffice to prove that given any dyadic grid $\mathcal{D}$,

$$
\left\|C_{b}^{\mathcal{D}} f\right\|_{L^{q}\left(w^{q}\right)} \leq C\|f\|_{L^{p}\left(w^{p}\right)},
$$

where $f$ is non-negative and in $L_{c}^{\infty}$. By duality there exists a non-negative function $g \in L^{q^{\prime}}\left(w^{-q}\right),\|g\|_{L^{q^{\prime}}\left(w^{-q}\right)}=1$, such that

$$
\begin{aligned}
\left\|C_{b}^{\mathcal{D}} f\right\|_{L^{q}\left(w^{q}\right)} & =\int_{\mathbb{R}^{n}} C_{b}^{\mathcal{D}} f(x) g(x) d x \\
& =\sum_{Q \in \mathcal{D}}|Q|^{\frac{\alpha}{n}} \int_{Q} f_{Q}|b(x)-b(y)| f(y) g(x) d y d x \\
& \leq \sum_{Q \in \mathcal{D}}|Q|^{\frac{\alpha}{n}} f_{Q}\left|b(y)-\langle b\rangle_{Q}\right| f(y) d y \int_{Q} g(x) d x \\
& \quad+\sum_{Q \in \mathcal{D}}|Q|^{\frac{\alpha}{n}} f_{Q}\left|b(x)-\langle b\rangle_{Q}\right| g(x) d x \int_{Q} f(y) d y \\
& =I_{1}+I_{2} .
\end{aligned}
$$

We will first estimate $I_{1}$. Let $v=w^{q}$ and $\sigma=w^{-p^{\prime}}$, and let $\Phi(t)=t \log (e+t)$. Then by Lemma 5.1 , since $[\sigma]_{A_{\infty}} \leq[w]_{A_{p, q}}^{p^{\prime}}$,

$$
\begin{aligned}
I_{1} & =\sum_{Q \in \mathcal{D}}|Q|^{\frac{\alpha}{n}} \sigma(Q) f_{Q}\left|b(y)-\langle b\rangle_{Q}\right| f(y) \sigma(y)^{-1} d \sigma f_{Q} g(x) d x \\
& \leq C \sum_{Q \in \mathcal{D}}|Q|^{\frac{\alpha}{n}} \sigma(Q)\left\|f \sigma^{-1}\right\|_{\Phi, Q, \sigma}\left\|b-\langle b\rangle_{Q}\right\|_{\bar{\Phi}, Q, \sigma} f_{Q} g(x) d x \\
& \leq C[w]_{A_{p, q}}^{p^{\prime}}\|b\|_{B M O} \sum_{Q \in \mathcal{D}}|Q|^{\frac{\alpha}{n}} \sigma(Q)\left\|f \sigma^{-1}\right\|_{\Phi, Q, \sigma} f_{Q} g(x) d x .
\end{aligned}
$$

We now want to show that we can replace the summation over cubes in $\mathcal{D}$ by a summation over a sparse subset $\mathcal{S}$ of $\mathcal{D}$. We do this using an argument from [7]; see also [9]. Fix $a=2^{n+1}$ and define the sets

$$
\Omega_{k}=\left\{x \in \mathbb{R}^{n}: M^{\mathcal{D}} g(x)>a^{k}\right\} .
$$

Then arguing exactly as in the construction of the Calderón-Zygmund cubes (see [8, Appendix A]), each set $\Omega_{k}$ is the union of a collection $\mathcal{S}_{k}$ of maximal, disjoint cubes in $\mathcal{D}$ that have the property that $a^{k}<\langle g\rangle_{Q} \leq 2^{n} a^{k}$. Moreover, the set $\mathcal{S}=\bigcup_{k} \mathcal{S}_{k}$ is sparse.

Now let

$$
\mathcal{C}_{k}=\left\{Q \in \mathcal{D}: a^{k}<\langle g\rangle_{Q} \leq a^{k+1}\right\}
$$


Then by the maximality of the cubes in $\mathcal{S}_{k}$, every cube $P \in \mathcal{C}_{k}$ is contained in a unique cube in $\mathcal{S}_{k}$. Therefore, we can continue the above estimate and apply Lemma 5.2 to get

$$
\begin{aligned}
I_{1} & \leq C[w]_{A_{p, q}}^{p^{\prime}}\|b\|_{B M O} \sum_{k} \sum_{Q \in \mathcal{C}_{k}}|Q|^{\frac{\alpha}{n}} \sigma(Q)\left\|f \sigma^{-1}\right\|_{\Phi, Q, \sigma} f_{Q} g(x) d x \\
& \leq C[w]_{A_{p, q}}^{p^{\prime}}\|b\|_{B M O} \sum_{k} a^{k} \sum_{P \in \mathcal{S}_{k}} \sum_{Q \subset P}|Q|^{\frac{\alpha}{n}} \sigma(Q)\left\|f \sigma^{-1}\right\|_{\Phi, Q, \sigma} \\
& \leq C[w]_{A_{p, q}}^{p^{\prime}}\|b\|_{B M O} \sum_{k} \sum_{P \in \mathcal{S}_{k}}|P|^{\frac{\alpha}{n}} \sigma(P)\left\|f \sigma^{-1}\right\|_{\Phi, P, \sigma} f_{P} g(x) d x \\
& =C[w]_{A_{p, q}}^{p^{\prime}}\|b\|_{B M O} \sum_{P \in \mathcal{S}}|P|^{\frac{\alpha}{n}-1} \sigma(P) v(P)^{1-\frac{\alpha}{n}}\left\|f \sigma^{-1}\right\|_{\Phi, Q, \sigma} v(P)^{\frac{\alpha}{n}} f_{Q} g v^{-1} d v .
\end{aligned}
$$

We can now argue exactly as in the proof of Theorem 1.2, beginning with the estimate (4.1) and applying Lemma 2.6 to complete the estimate of $I_{1}$ with a constant $[w]_{A_{p, q}}^{p^{\prime}+1+\frac{q}{p^{\prime}}+\frac{p^{\prime}}{p}}$.

The estimate for $I_{2}$ is essentially the same, exchanging the roles of $f$ and $g$ and $\sigma$

and $v$. This yields the above estimate except that the constant is now $[w]_{A_{p, q}}^{q+1+\frac{q}{p^{\prime}}+\frac{p^{\prime}}{p}}$. This completes the proof.

\section{Tres Recuerdos de Cora Sadosky}

I first met Cora at an AMS sectional meeting in Burlington, Vermont, in 1995. It began inauspiciously: at the reception on the first night, a determined looking woman came up to me, waved her finger under my nose and said, "I have a bone to pick with you. We will talk later," and then marched off. She found me again about 30 minutes later and proceeded to explain. The year before she had been asked by an NSF reviewer for her opinion of my proposal which mentioned the two weight problem for the Hilbert transform. She had told the reviewer to refer me to a paper by her and Mischa Cotlar where they gave the first (and for a long time the only) characterization of these pairs of weights. He did not, however, share this reference, and when I published the paper based on this work I did not cite it. Cora assumed that I had simply disregarded this advice and was understandably annoyed. However, once I explained that I had never received this information she immediately became much friendlier and invited me to visit her in Washington, D.C.

For the next few years she took an interest in my career. Her first major intervention on my behalf came in 1996, when she applied her forceful personality, first to convince me that I must attend the El Escorial conference in 1996 (despite moving, changing jobs, and having two small children and a pregnant wife), and then to 
strong-arm funding from a colleague to pay for my trip. It was at this meeting that I met, among others, Carlos Pérez, and began a collaboration that has continued to the present day.

Two years later, in 1998, we met again at an AMS sectional meeting in Albuquerque. At this meeting she picked up on a point that my Spanish colleagues were also making: given my name and my ancestry, I really ought to be able to speak Spanish. Her solution was that I should "read a good math book in Spanish." She strongly recommended that I read Javier Duoandikoetxea's book, Análisis de Fourier, telling me that I would see some good mathematics as well as "learn Spanish." For the next year I worked through the text line by line, in the process writing a complete translation. I approached Javi with an offer to complete the translation and update the notes, and together we produced an English edition. In the process I did in fact learn a great deal of harmonic analysis, but unfortunately, Cora's original goal was not achieved: my spoken Spanish did not improve appreciably. Moreover, this translation had the unintended consequence of convincing large numbers of mathematicians from Spain, Argentina and elsewhere that I did in fact speak Spanish.

Cora and I never collaborated on a paper. She suggested several projects, but my interests were moving away from hers and nothing came to fruition. At the time I never really quite understood or appreciated the support she provided at these points in my career, and it is only in looking back that I realize how much I owe her. So belatedly I say, muchísimas gracias, Cora.

\section{REFERENCES}

[1] L. Chaffee. Characterizations of BMO through commutators of bilinear singular integral operators. preprint, October 2014. ArXiv 1410.4587.

[2] S. Chanillo. A note on commutators. Indiana Univ. Math. J., 31(1):7-16, 1982.

[3] M. Christ and R. Fefferman. A note on weighted norm inequalities for the Hardy-Littlewood maximal operator. Proc. Amer. Math. Soc., 87(3):447-448, 1983.

[4] D. Chung, C. Pereyra, and C. Pérez. Sharp bounds for general commutators on weighted Lebesgue spaces. Trans. Amer. Math. Soc., 364(3):1163-1177, 2012.

[5] D. Cruz-Uribe. Two weight norm inequalities for fractional integral operators and commutators. preprint, 2015. ArXiv 1412.4157.

[6] D. Cruz-Uribe and A. Fiorenza. Endpoint estimates and weighted norm inequalities for commutators of fractional integrals. Publ. Mat., 47(1):103-131, 2003.

[7] D. Cruz-Uribe, J. M. Martell, and C. Pérez. Extensions of Rubio de Francia's extrapolation theorem. Collect. Math., (Vol. Extra):195-231, 2006.

[8] D. Cruz-Uribe, J. M. Martell, and C. Pérez. Weights, extrapolation and the theory of Rubio de Francia, volume 215 of Operator Theory: Advances and Applications. Birkhäuser/Springer Basel AG, Basel, 2011.

[9] D. Cruz-Uribe and K. Moen. Sharp norm inequalities for commutators of classical operators. Publ. Mat., 56:147-190, 2012.

[10] D. Cruz-Uribe and K. Moen. A fractional Muckenhoupt-Wheeden theorem and its consequences. Integral Equations Operator Theory, 76(3):421-446, 2013. 
[11] D. Cruz-Uribe and K. Moen. One and two weight norm inequalities for Riesz potentials. Illinois J. Math., 57(1):295-323, 2013.

[12] J. Duoandikoetxea. Fourier analysis, volume 29 of Graduate Studies in Mathematics. American Mathematical Society, Providence, RI, 2001.

[13] J. Duoandikoetxea, F. J. Martín-Reyes, and S. Ombrosi. On the $A_{\infty}$ conditions for general bases. preprint, 2013.

[14] K.-P. Ho. Characterizations of BMO by $A_{p}$ weights and p-convexity. Hiroshima Math. J., 41(2):153-165, 2011.

[15] T. Hytönen. The $A_{2}$ theorem: Remarks and complements. preprint, 2012. ArXiv 1212.3840.

[16] T. Hytönen and C. Pérez. Sharp weighted bounds involving $A_{\infty}$. Anal. PDE, 6(4):777-818, 2013.

[17] M. T. Lacey, K. Moen, C. Pérez, and R. H. Torres. Sharp weighted bounds for fractional integral operators. J. Funct. Anal., 259(5):1073-1097, 2010.

[18] A. K. Lerner. A simple proof of the $A_{2}$ conjecture. Int. Math. Res. Not. IMRN, (14):3159-3170, 2013.

[19] B. Muckenhoupt and R. L. Wheeden. Weighted norm inequalities for fractional integrals. Trans. Amer. Math. Soc., 192:261-274, 1974.

[20] C. Pérez. Two weighted inequalities for potential and fractional type maximal operators. Indiana Univ. Math. J., 43(2):663-683, 1994.

[21] M. M. Rao and Z. D. Ren. Theory of Orlicz spaces, volume 146 of Monographs and Textbooks in Pure and Applied Mathematics. Marcel Dekker Inc., New York, 1991.

[22] E. T. Sawyer. A two weight weak type inequality for fractional integrals. Trans. Amer. Math. Soc., 281(1):339-345, 1984.

[23] E. T. Sawyer and R. L. Wheeden. Weighted inequalities for fractional integrals on Euclidean and homogeneous spaces. Amer. J. Math., 114(4):813-874, 1992.

[24] E. M. Stein. Singular integrals and differentiability properties of functions. Princeton Mathematical Series, No. 30. Princeton University Press, Princeton, N.J., 1970.

[25] E. M. Stein and G. Weiss. Introduction to Fourier analysis on Euclidean spaces. Princeton University Press, Princeton, N.J., 1971. Princeton Mathematical Series, No. 32.

Department of Mathematics, Trinity College, Hartford, CT 06106, USA

E-mail address: david.cruzuribe@trincoll.edu 